\title{
A Systematic Review and Meta-Analysis of Anxiety among Children and Youth in Ethiopia
}

\author{
Berhanu Boru Bifftu ${ }^{*}$ and Yonas Deressa Guracho ${ }^{2}$ \\ ${ }^{1}$ School of Nursing, University of Gondar College of Medicine and Health Science, Gondar, Ethiopia \\ ${ }^{2}$ Department of Psychiatry, College of Medicine and Health Science, Bahar Dar University, Ethiopia
}

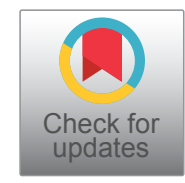

\begin{abstract}
Background: Anxiety disorders are the most common mental illness that has an immense impact on the life of children and youth. Thus, the present study aimed to determine the pooled prevalence of depression among children and youth in Ethiopia.

Methods: A compressive search of electronic databases including: PubMed/Medline, CINAHL (EBSCOhost), SCOPUS, African Journal Online (AJOL) and African Indexed Medicus (AIM) were searched. Heterogeneity test was assessed by Cochrane chi-square $\left(\chi^{2}\right)$ and quantified by $\mathrm{I}^{2}$ statistics test. Sensitivity test and subgroup analysis were performed. Publication bias was determined by funnel plots and Egger's test.

Results: In this study, six different types of anxiety were included from a total of 16 studies. The prevalence of anxiety disorders was ranged from $0.03 \%$ (post-traumatic disorder) to $63 \%$ (social phobia). The ranged prevalence of GAD, phobia, social phobia, separation anxiety, OCD and PTSD was 0.5-23\%, 0.7-27\%, 4.3-63\%, 0.4-17.7\%, 0.1-67, and 0.03-1.2 respectively. The overall pooled prevalence of anxiety disorder was $8 \%\left[95 \% \mathrm{Cl}: 4 \%\right.$ to $14 \%, \mathrm{I}^{2}=98.36 \%, \mathrm{p}<0.001$ (Figure $1)]$. The overall test of heterogeneity showed significant heterogeneity $\left[I^{2}=98.36 \%, p<0.001\right.$; however, sensitivity analysis showed none of influential individual study on the overall effect and no evidence of publication bias from funnel plot and Egger's test $(\mathrm{P}<0.363)$.
\end{abstract}

Conclusion: Overall, one in twelve children and youth had anxiety. This suggests the needs of children and youth targeted mental health screening and care. Further research is needed to identify contextual risk and protective factors for mental health promotion, prevention and treatment.

\section{Keywords}

Anxiety, Children, Ethiopia, Young people

\section{Background}

Child and adolescent mental health is the capacity to achieve and maintain optimal psychological well being $[1,2]$. Globally, $10 \%$ to $20 \%$ of children and adolescents have mental health problems [1-5]. Anxiety is a normal, emotional, reasonable and expected response to real or potential danger; however, it is a pathological when symptoms are prolonged, irrational, in the absence of stimuli and interfere with functionality $[6,7]$. Pathologic anxiety is characterized by emotional feelings of anxiety, fear and physical symptoms such as increased blood pressure, quickened respiration and tightness of the chest $[2,7,8]$. In the clinical psychopathology, the term "anxiety" refers to a subjective feeling of unease, discomfort, apprehension or fearful concern accompanied by a host of autonomic and somatic manifestations [7-9]. Anxiety disorders are among the most common mental illness with estimated prevalence of 10.6-24.3, 16.6-41 in the adult general population [7,9-14]. Childhood and adolescence are crucial developmental periods in terms of brain structures and neurons systems maturing, which has implications for psychosocial well-being in cognitive, emotion, motivation and social functioning. Evidence showed that nearly one in two adult mental health problems were begin by the age of 14 and the remaining $75 \%$ by the mid-20s $[7,15,16]$. Early onset of mental illness associated with chronic course, co-morbid,

*Corresponding author: Berhanu Boru Bifftu, School of Nursing, University of Gondar College of Medicine and Health Science, Gondar, Ethiopia, Tel: +251-911-53-59-70

Accepted: July 03, 2021

Published online: July 05, 2021

Citation: Bifftu BB, Guracho YD (2021) A Systematic Review and Meta-Analysis of Anxiety among Children and Youth in Ethiopia. J Depress Anxiety Disord 3(2):89-100 
substance use, suicide and variety of negative outcomes including: lower educational achievement, impaired functioning and poorer quality of life $[1,7,15]$. A number of community based systematic review and meta-analysis were conducted in every region of the world indicated range of $6.5 \%$ to $10 \%$ pooled prevalence for any anxiety disorders in children and youth $[1,4,10,17,18]$. Several complex interactions of risk factors including children characteristics [gender, age, ethnicity, physical health, function, exposures to illness, stress, substance, infections], parent characteristics including: Parental education, age, social class, employment, psychiatric and medical history, and family function, structure and neighborhood and contextual related characteristics were associated with anxiety disorders in children and adolescent $[1,4,7,8,10,19]$. The needs of culture specific epidemiological evidences for children and youth mental health have several reasons: (i) Substantial proportion of mental disorders (50\%) begins by the age of 15 and $75 \%$ by the age of 25), (ii) High degree of continuity between child, adolescent and adulthood, (iii) The growing political interest in child and adolescent mental health, (iv) Children and youth are future asset of the society. Despite its early onset, chronic course, adverse effects and epidemiological variation across the world, there is little culture specific evidence of systematic review and meta-analysis in several developing countries including Ethiopia. In Ethiopia, mental illness account for $11 \%$ of the total burden of disease. Depression, psychosis, bipolar disorder, epilepsy, developmental disorders, behavioral disorders, dementia, alcohol use and alcohol use disorders, drug use, self-harm and suicide and other emotional or medically unexplained complaints are the country's identified priority area in mental health $[20,21]$. Of these, anxiety is one of the most commonly identified priority mental illness with an estimated prevalence of $3.3 \%$ and accounting for $8.5 \%$ of all years lived with disability in the general population [11]. In children and young people, $50 \%$ of the populations, the prevalence of any mental disorder was ranged from $12-25 \%$ and $0.03-63 \%$ for anxiety $[3,22-35]$. The presence of mental illness particularly anxiety disorders in children and youth associated with higher unemployment, poverty, lower educational attainment, poorer physical health, suicide, persist and continue to adulthood and increased mortality $[31,32,36]$. In addition to these, children and youth population in the country have no distinct category of mental health services for children. However, in the recently planned scale-up of mental health services $[20,37,38]$, children and adolescents are identified as a vulnerable group that deserve special attention [21]. Despite its early age of onset, chronic course, high prevalence, treatment gap, its associated impact, several inconsistent individual studies, there is no national level representative evidence for children and youth in Ethiopia. Thus, the present systematic review and meta-analysis aimed to determine the pooled prevalence of anxiety among children and youth in the general populations of Ethiopia.

\section{Methods}

\section{Search strategy}

Initially, the Database of Abstracts of Reviews of Effects (DARE) and the Cochrane Database of Systematic Reviews
(CDSR) were searched to ensure the absence of similar studies in Ethiopia. Then the search strategy conducted using: First a compressive search of electronic databases including: PubMed/Medline, CINAHL (EBSCOhost), SCOPUS, African Journal Online (AJOL) and African Indexed Medicus (AIM). Keywords, Medical Subject Headings (MeSH) terms and Boolean operators ("AND" and/“OR") were established for each databases. The second stage of search strategy involved scanning relevant websites, including national and international institutions and research centers, web of science (Google and Google Scholars) for grey literatures. Finally, reference lists of included articles were searched. All searches were performed until April, 17, 2021. The full electronic search strategy for PubMed/MEDLINE database: ((school ${ }^{*}$ [MeSH Terms]) OR (school $\left.{ }^{*}\right)$ OR (adolescen ${ }^{*}$ [MeSH Terms]) OR (adolescen $\left.{ }^{*}\right)$ OR (child* [MeSH Terms]) OR (child*) OR (child [MeSH Terms]) OR (child) OR (youth [MeSH Terms]) OR (youth) OR (teen [MeSH Terms]) OR (teen) OR (teenagers [MeSH Terms]) OR (teenagers) OR (community [MeSH Terms]) OR (community) OR (students [MeSH Terms]) OR (students)) AND ((anxiety [MeSH Terms]) OR (anxiety) OR anxiety symptoms [MeSH Terms]) OR (anxiety symptoms) OR anxiety disorder [MeSH Terms]) OR (anxiety disorder) OR (generalized anxiety disorder [MeSH Terms]) OR (generalized anxiety disorder) OR (fear [MeSH Terms]) OR (fear) OR (phobia [MeSH Terms]) OR (phobia) OR (social phobia [MeSH Terms]) OR (social phobia) OR (social anxiety [MeSH Terms]) OR (social anxiety) OR (agoraphobia [MeSH Terms]) OR (agoraphobia) OR (specific phobia [MeSH Terms]) OR (specific phobia) OR (separation anxiety [MeSH Terms]) OR (separation anxiety) OR (panic disorder [MeSH Terms]) OR (panic disorder) OR (overanxious disorder [MeSH Terms]) OR (overanxious disorder) OR (post-traumatic stress disorder [MeSH Terms]) OR (post-traumatic stress disorder) OR (obsessive compulsive disorder [MeSH Terms]) OR (obsessive compulsive disorder) OR (obsession [MeSH Terms]) OR (obsession) OR (compulsion [MeSH Terms]) OR (compulsion)) AND ((epidemiology [MeSH Terms]) OR (epidemiology) OR (prevalence [MeSH Terms]) OR (prevalence) OR (incidence [MeSH Terms]) OR (incidence)) AND Ethiopia.

Similarly, CINAHL (EBSCO host) and AJOL databases were searched using similar search terms tailored to each database. The report of this systematic review and metaanalysis was conducted according to the Preferred Reporting Items for Systematic Review and Meta-Analysis (PRISMA) (Supplement) [39].

\section{Selection of studies}

All studies retrieved through search strategy were imported in to EndNote X7 (Thomson Reuters, New York, USA) and removed duplicated studies. The study selection process had three stages: The first stage was screened based on the title and abstract of retrieved references against the outcome. The second stage was based on the full-text to determine its applicability to the study aim. Finally, studies were screened based on the eligibility criteria. Two reviewers (YDG and BBB) independently screened the studies. Disagreements were solved by discussion. 


\section{Definition of concepts}

For the purpose of this study, we defined anxiety disorders as the presence of combination of any of the specific type of anxiety disorders including: Generalized anxiety disorder/ overanxious, agoraphobia, social phobia/social anxiety disorder, simple/isolated/specific phobia, post-traumatic stress disorder, separation anxiety disorder and obsessivecompulsive disorder. A study had to report the combined overall prevalence or at least two anxiety disorders for meta-analysis. For those studies reported multiple separate outcomes, average combined effect sizes was computed for the pooled meta-analysis of overall anxiety. Although there have been changes in the criteria of anxiety disorders in the Diagnostic and Statistical Manual of Mental Disorders (Fifth Edition: DSM-V), studies presented in this review were reported based on DSM-IV and ICD-10; therefore, OCD and PTSD included. For the assessment of anxiety disorder/ symptoms, both diagnostic interviews [include: CIDI, the Revised parent version of the Diagnostic Interview for Children and Adolescents (DICA-R); $n=1$ ), Diagnostic and Statically Manual of Mental disorder third and fourth edition (DSM-III-R and IV)] and self-report rating scales [include: Child Behavior check list $(\mathrm{CBCL})$, Screen for Child Anxiety Related Emotional Disorders (SCARED), HADS, CBCL, ASEBA, HRSRS) and statement: "During the past 12 months, how often have you been so worried about some ting that you could not sleep at night?].

A child is defined as an individual under the age of 18 years [40]. According to the World Health Organization (WHO) recognizes persons aged 10-19 as adolescents, aged 15-24 as youth and a comprehensive category of ages 10-24 as young [18]. In this study, children and young people are those participants with age $\leq 24$ years.

\section{Eligibility Criteria}

According to the PICOS acronym, the following inclusion criteria were used: Participants $(P)$ : Children and youth age $\leq$ 24 years. Studies were considered if the prevalence of anxiety was reported separately for participants of age $\leq 24$ years. There was no minimum age limited. Intervention (I): Not applicable; Comparison (C): Not applicable; Outcomes (O): Prevalence of anxiety; Study design (S): Observational studies [cross-sectional, case-control and cohort/longitudinal] and conducted in Ethiopia. Exclusion criteria: (1) Case reports, conference, reviews and abstracts; and (2) Studies conducted among university students, clinical population (e.g. psychiatric, physical problems including HIV/AIDs) and specific group such as: refugees and street (homeless) and (3) Special cases of anxiety are secondary anxiety, such as substance-induced anxiety disorder and anxiety disorder due to medical condition.

\section{Data extraction}

A pre-conceived and piloted data extraction Microsoft Excel format was used by two independent reviewers (YDG and $\mathrm{BBB}$ ). The extracted data include: name of the first author, year of publication, study setting, study population, age of participants (mean age or range), assessment tool, sample size and number of cases/prevalence of anxiety.

\section{Quality assessment}

We used the Newcastle-Ottawa quality assessment scale adapted for cross-sectional studies under three main parts (selection, comparability and outcome) [41]. The scale is used to score the articles under three categories: (i) Selection (score 0-5); (ii) Comparability (score 0-2); and (iii) Outcome (score 0-3). The selection part assesses the methodological quality of: Representativeness of the sample, adequacy of the sample size, response rate and use of validated measurement tool to gather data on exposure. The comparability category examines whether the subjects are comparable in different outcome groups based on the study design, analysis and confounding factors were controlled or not. The outcome part assessed whether outcome(s) used independent blind assessment, records or self-reporting assessment. The outcome part also assessed whether the statistical tests used to analyze data were clearly described and appropriate. The quality of each study was determined by the summed score of all items under the three parts to generate a study specific overall score. Individual paper was graded with score ranged from zero to ten stars and categorized as good quality for scored $\geq 7$ stars, medium (4-6) and poor quality for scored $\leq 3$.

\section{Coding of Studies}

Studies were coded using a pre-formulated coding sheet according to: author name, year of published, sample size ( $\geq$ median vs. < median), study setting, population source (general population and students), anxiety measurement tool and quality of study (good vs. fair).

\section{Data synthesis and statistical analysis}

The extracted data were exported from Microsoft Excel Database to Stata version 14 that we installed packages for meta-analyses. Separate meta-analyses were performed for anxiety disorders with at least two studies using Metaprop command in Stata employed to calculate the pooled prevalence at $95 \% \mathrm{Cls}$ by a random-effects model [42]. Test for Heterogeneity was performed using Cochrane chi-square $\left(\chi^{2}\right)$ and the $I^{2}$ statistics. $I^{2}$ value greater than $50 \%$ was considered as indicative of substantial heterogeneity [43]. P-value < 0.10 was considered as indicative of statistically significant heterogeneity. To assess the possible source of heterogeneity, a sensitivity analysis was performed to assess whether the pooled prevalence estimates of anxiety was influenced by individual studies [44]. In addition to this, subgroup analysis was conducted by sample size, study setting, publication years, assessment tool and quality of study. Publication bias was tested by the visual inspection of funnel plot [45] and egger test [46]. A p-value $<0.1$ was considered as indicative of statistically significant publication bias. The findings of this study summarized and presented using texts, forest plots, tables and summery of descriptive statistics.

\section{Results}

The initial database search resulted in 2414 publications. Additional, 17 studies were located from the other sources. This resulted in 2431 records. Of these records, 1278 were excluded because of duplication. Of the remaining 1153, 752 
Citation: Bifftu BB, Guracho YD (2021) A Systematic Review and Meta-Analysis of Anxiety among Children and Youth in Ethiopia. J Depress Anxiety Disord 3(2):89-100

studies excluded as their title and abstract reading was not related to the outcome. From the remaining 401 studies, 209 articles were excluded based on the full text/information and found unclear results. Finally, 176 publications were excluded based on the eligibility criteria and the remained 16 studies included in the systematic review and meta-analysis (Figure 2).

\section{Study characteristics}

In this study, six different types of anxiety disorders were included from a total of 16 studies [GAD $(n=7)$, phobia $(n=$ $6)$, social phobia $(n=2)$, separation anxiety $(n=3)$, OCD $(n=$ $2)$, PTSD $(n=3)$ and 11 for overall anxiety] were included in this systematic review and meta-analysis. These studies were carried out in five different regions of the country: Oromia ( $\mathrm{n}$ $=4)$, Amhara $(n=2)$, Addis Ababa $(n=5)$, SNNP $(n=4)$ and Tigray $(n=1)$. These studies were published between 1991 and 2019. All studies used cross-sectional study design. The sample sizes of included studies ranged from 112 to 2401. For the assessment of anxiety, both diagnostic interviews [include: Composite International Diagnostic Interview (CIDI), the Revised parent version of the Diagnostic Interview for Children and Adolescents (DICA-R); $n=1$ ), Diagnostic and Statically Manual of Mental disorder third and fourth edition (DSM-III-R and IV) and self-report rating scales [include: Child Behavior check list (CBCL)], Screen for Child Anxiety Related Emotional Disorders (SCARED), Hospital Anxiety and Depression Scale (HADS), Achenbach System of Empirically Based Assessment (ASEBA), Holmes-Rahe Social Re-adjustment Scale (HRSRS)] and using the item "During the past 12 months, how often have you been so worried about some ting that you could not sleep at night? (Table 1).

\section{Quality of included studies}

The quality of included articles in this systematic review and meta-analysis is shown in Table 2. The highest scored quality items in the observed studies were the representativeness of sample, use of validated measurement tool for the assessment of anxiety and reported appropriate statistical analysis by all studies (100\%); while, the sample size $(54 \%)$ and response rate (69\%) were the list observed scored quality items. The overall quality score of individual study ranged from six to eight. Of this ten articles (54\%) were good quality and three studies were fair quality.

\section{Type and prevalence of anxiety disorders}

In this study, six different types of anxiety disorders were identified: $\operatorname{GAD}(n=7)$, phobia $(n=6)$, social phobia $(n=2)$, separation anxiety $(n=3), \operatorname{OCD}(n=2)$ and PTSD ( $=3$ ). In addition to these, 11 studies were included in the overall anxiety disorders (Table 2 ). The prevalence of anxiety disorders was ranged from $0.03 \%$ (post traumatic disorder) to $63 \%$ (social phobia) (Table 1). The ranged prevalence of GAD, phobia, social phobia, separation anxiety, OCD and PTSD was $0.5-23 \%, 0.7-27 \%, 4.3-63 \%, 0.4-17.7 \%, 0.1-67 \%$, and $0.03-1.2 \%$ respectively. The corresponding figures for the estimated pooled prevalence of GAD, phobia, social phobia, separation anxiety, OCD and PTSD was $11 \%$ [95\% Cl: 5-19\%], $7 \%$ [95\%Cl: 2-14\%], 31\% [95\%Cl: 27-35\%], 4\% [95\%Cl: 0-15\%],

Table 1: Characteristics, type and prevalence of anxiety disorder in the included studies.

\begin{tabular}{|c|c|c|c|c|c|c|c|c|c|c|c|c|c|c|c|c|c|}
\hline \multirow[t]{2}{*}{ Author, year } & \multirow{2}{*}{$\begin{array}{l}\text { Age } \\
\text { range }\end{array}$} & \multirow{2}{*}{$\begin{array}{c}\text { Assessment } \\
\text { tool }\end{array}$} & \multirow{2}{*}{$\begin{array}{l}\text { Study } \\
\text { sample }\end{array}$} & \multicolumn{2}{|c|}{ Any ADs } & \multicolumn{2}{|c|}{ GAD } & \multicolumn{2}{|c|}{ Phobia } & \multicolumn{2}{|c|}{$\begin{array}{l}\text { Social } \\
\text { phobia }\end{array}$} & \multicolumn{2}{|c|}{$\begin{array}{c}\text { Separation } \\
\text { anxiety }\end{array}$} & \multicolumn{2}{|c|}{ PTSD } & \multicolumn{2}{|c|}{ OCD } \\
\hline & & & & event & $\%$ & event & $\%$ & event & $\%$ & event & $\%$ & event & $\%$ & event & $\%$ & event & $\%$ \\
\hline Achiko, 2019 & $12-17$ & SCARED & 299 & - & - & - & - & - & - & 189 & 63 & - & - & - & - & - & - \\
\hline $\begin{array}{l}\text { Asfawesen, } \\
2018\end{array}$ & $10-14$ & HADS & 297 & - & - & 52 & 18 & - & - & - & - & - & - & - & - & - & - \\
\hline Fekadu, 2006 & $5-15$ & DSM-III-R & 472 & 12 & 2.5 & - & - & - & - & - & - & 7 & 1.5 & - & - & - & - \\
\hline Kebede, 1997 & $6-18$ & DICA-R & 255 & 153 & 2 & 29 & 11.4 & 68 & 26.7 & 11 & 4.3 & 45 & 17.7 & 3 & 1.2 & 16 & 6.3 \\
\hline Ashenafi, 2001 & $5-15$ & DSM-II-R & 1477 & 24 & 5 & 7 & 0.5 & 11 & 0.7 & - & - & - & - & 9 & 0.6 & - & - \\
\hline Mulatu, 1999 & $6-11$ & $\mathrm{CBCL}$ & 611 & 29 & 4.7 & - & - & - & - & - & - & - & - & - & - & - & - \\
\hline Desta, 2008 & $6-15$ & DICA-R & 668 & 11 & 1.6 & - & - & - & - & - & - & 3 & 0.4 & 1 & 0.03 & 1 & 0.1 \\
\hline Alem, 2006 & $8-15$ & DICA-R & 400 & 12 & 3 & - & - & 21 & 5.3 & - & - & 2 & 0.5 & - & - & - & - \\
\hline Nyundo, 2019 & $10-19$ & - & 1059 & - & - & 132 & 12.5 & - & - & - & - & - & - & - & - & - & - \\
\hline Nyundo, 2019 & $10-19$ & - & 951 & - & - & 84 & 9 & - & - & - & - & - & - & - & - & - & - \\
\hline Asnake, 2016 & $15-24$ & ASEBA & 403 & 42 & 10.4 & - & - & - & - & - & - & - & - & - & - & - & - \\
\hline Awas, 1999 & $15-24$ & CIDI & 112 & 8 & 7 & - & - & 7 & 6 & - & - & - & - & - & - & - & - \\
\hline Kebede, 2005 & $15-24$ & CIDI & 613 & 65 & 11 & - & - & - & - & - & - & - & - & - & - & - & - \\
\hline $\begin{array}{l}\text { Wendemagegn, } \\
2017\end{array}$ & $15-24$ & DSM-IV & 215 & - & - & 50 & 23 & - & - & - & - & - & - & - & - & - & - \\
\hline Kebede, 1999 & $15-24$ & CIDI & 529 & 40 & 8 & 53 & 10 & 27 & 5 & - & - & - & - & - & - & - & - \\
\hline Tafari, 1991 & $15-24$ & HRSRS & 418 & 21 & 5 & - & - & - & - & - & - & - & - & - & - & - & - \\
\hline
\end{tabular}

Note: ASEBA: Achenbach System of Empirically Based Assessment; CBCL: Child Behaviour Checklist; CIDI: Composite International Diagnostic Interview; DICA-R: Diagnostic Interview for Children and Adolescent; DSM-IV: Diagnostic and Statistical Manual for mental health $4^{\text {th }}$ Edition; HADS: Hospital Anxiety and Depression Scale; HRSRS: Holmes-Rahe Social Readjustment Scale; SCARED: Screen for Child Anxiety Related Emotional Disorders. 
Citation: Bifftu BB, Guracho YD (2021) A Systematic Review and Meta-Analysis of Anxiety among Children and Youth in Ethiopia. J Depress Anxiety Disord 3(2):89-100

Table 2: Quality of included studies in the analysis $(n=16)$.

\begin{tabular}{|l|c|c|c|c|c|c|c|}
\hline Author, year & \multicolumn{9}{c}{ Quality domain } & \multicolumn{2}{c}{ Overall } \\
Score
\end{tabular}

Keys:

A. Q1-Q7 represents questions used to assess the quality of included studies.

Selection

Q1. Representativeness of the sample: a) Truly representative of the average in the target population* (all subjects or random sampling); b) Somewhat representative of the average in the target population* (non-random sampling); c) Selected group of users. d) No description of the sampling strategy.

Q2. Sample size: a) Justified \& satisfactory*, b) Not justified.

Q3. Non-respondents: a) Comparability between respondents and non-respondents characteristics is established, and the response rate is satisfactory*, b) The response rate is unsatisfactory, or the comparability between respondents and non-respondents is unsatisfactory, c) No description of the response rate or the characteristics of the responders and the non-responders.

Q4. Ascertainment of the exposure/risk factor: Confounding factors are controlled. a) The study controls for the most important factor (select one)* b) The study control for any additional factor*.

Comparability

Q5. The subjects in different outcome groups are comparable, based on the study design (e. g. case or cohort) or analysis. a) Validated measurement tool. **, b) Non-validated measurement tool, but the tool is available or described. ${ }^{*}$ c) No description of the measurement tool.

Outcome

Q6. Assessment of outcome: a) Independent blind assessment ** b) Record linkage** c) Self report * d) No description.

Q7. Statistical test: a) is clearly described, appropriate, \& measurement of association is presented, including confidence intervals \& probability level ( $p$ value)* $b$ ) is not appropriate.

$1 \%$ [95\% Cl: $0-2 \%]$ and $0 \%[95 \% \mathrm{Cl}: 0-2 \%]$ respectively (Figure 3)]. The overall pooled prevalence of anxiety disorder was $8 \%$ [95\% Cl: $4-14 \%, I^{2}=98.36 \%, p<0.001$ (Figure 1)]. The overall test for heterogeneity showed evidence of significant heterogeneity $\left[I^{2}=98.36 \%, p<0.001\right.$, (Figure 1 ) $]$. The subgroup analysis by study sample size, publication year, type of anxiety disorder and quality of study could not identified source of heterogeneity; however, the highest pooled prevalence was observed from studies carried out in Addis Ababa [13\% (2\%32\%)] (Table 3). The overall sensitivity analysis showed that none of the point estimates was outside of the overall $95 \% \mathrm{Cl}$ (Figure 4). In other words, no outliers (defined as studies from which the $95 \% \mathrm{Cl}$ does not overlap with the $95 \% \mathrm{Cl}$ of the pooled effect size). No evidence of publication bias from the visual inspection of the funnel plot (Figure 5) and [Egger's test $(P=0.363)]$. The number of included studies was too low to investigate publication bias, meta-regression or to perform sensitivity analyses for the categories of anxiety disorders.

\section{Discussion}

To the best of our knowledge, this meta-analysis is the first to report the prevalence of ADs and their subtypes in Ethiopia. The observed pooled prevalence of anxiety [8\% (95\% Cl: $4 \%$ to $14 \%)]$ in this study is higher than the reported national prevalence in adult (3.3\%). This showed that children and youth had high prevalence of anxiety as compared to the adult general population. This suggests the needs of evaluating the priority focus of the country's mental health strategy. In Ethiopia, though the Government identified children and adolescents as a vulnerable group for mental illness and planned to scale-up the limited mental health services [20,37]; practically, there is a huge gap in policy formulation and implementation at the grassroots level [21] as a results, children and adolescent were not considered as a unique population and served under a mental health services of the adult population. 
Citation: Bifftu BB, Guracho YD (2021) A Systematic Review and Meta-Analysis of Anxiety among Children and Youth in Ethiopia. J Depress Anxiety Disord 3(2):89-100

Table 3: Subgroup analyses of the included studies.

\begin{tabular}{|c|c|c|c|c|c|}
\hline Subgroup & $\begin{array}{l}\text { No. of } \\
\text { studies }\end{array}$ & Pooled prevalence & $95 \% \mathrm{Cl}$ & $I^{2}$ & P-value \\
\hline \multicolumn{6}{|l|}{ Sample size } \\
\hline Small (< median) & 6 & 4 & $2-8$ & $98.81 \%$ & $<0.001$ \\
\hline Large ( $\geq$ median) & 5 & 8 & $4-14$ & $97.54 \%$ & $<0.001$ \\
\hline \multicolumn{6}{|l|}{ Publication year } \\
\hline 1991-2005 & 6 & 11 & $3-25$ & $93.42 \%$ & $<0.001$ \\
\hline 2006-2016 & 5 & 5 & $2-9$ & $99.23 \%$ & $<0.001$ \\
\hline \multicolumn{6}{|l|}{ Tool } \\
\hline Screening & 3 & 9 & $3-16$ & $90.28 \%$ & $<0.001$ \\
\hline Diagnostic & 8 & 6 & $4-10$ & $98.94 \%$ & $<0.001$ \\
\hline \multicolumn{6}{|l|}{ Setting } \\
\hline Addis Ababa & 4 & 13 & $2-32$ & $98.95 \%$ & $<0.001$ \\
\hline Oromia & 2 & 2 & $2-3$ & -- & -- \\
\hline Amhara & 2 & 6 & $5-8$ & -- & -- \\
\hline SNNP & 3 & 7 & $4-12$ & -- & -- \\
\hline \multicolumn{6}{|l|}{ QOL } \\
\hline Good quality & 8 & 8 & $3-16$ & $99.28 \%$ & $<0.001$ \\
\hline Fair quality & 3 & 7 & $4-12$ & $78.98 \%$ & $<0.001$ \\
\hline
\end{tabular}

Note: $95 \% \mathrm{Cl}$ represents the $95 \%$ Confidence Interval for prevalence and $\mathrm{I}^{2}$ true heterogeneity.

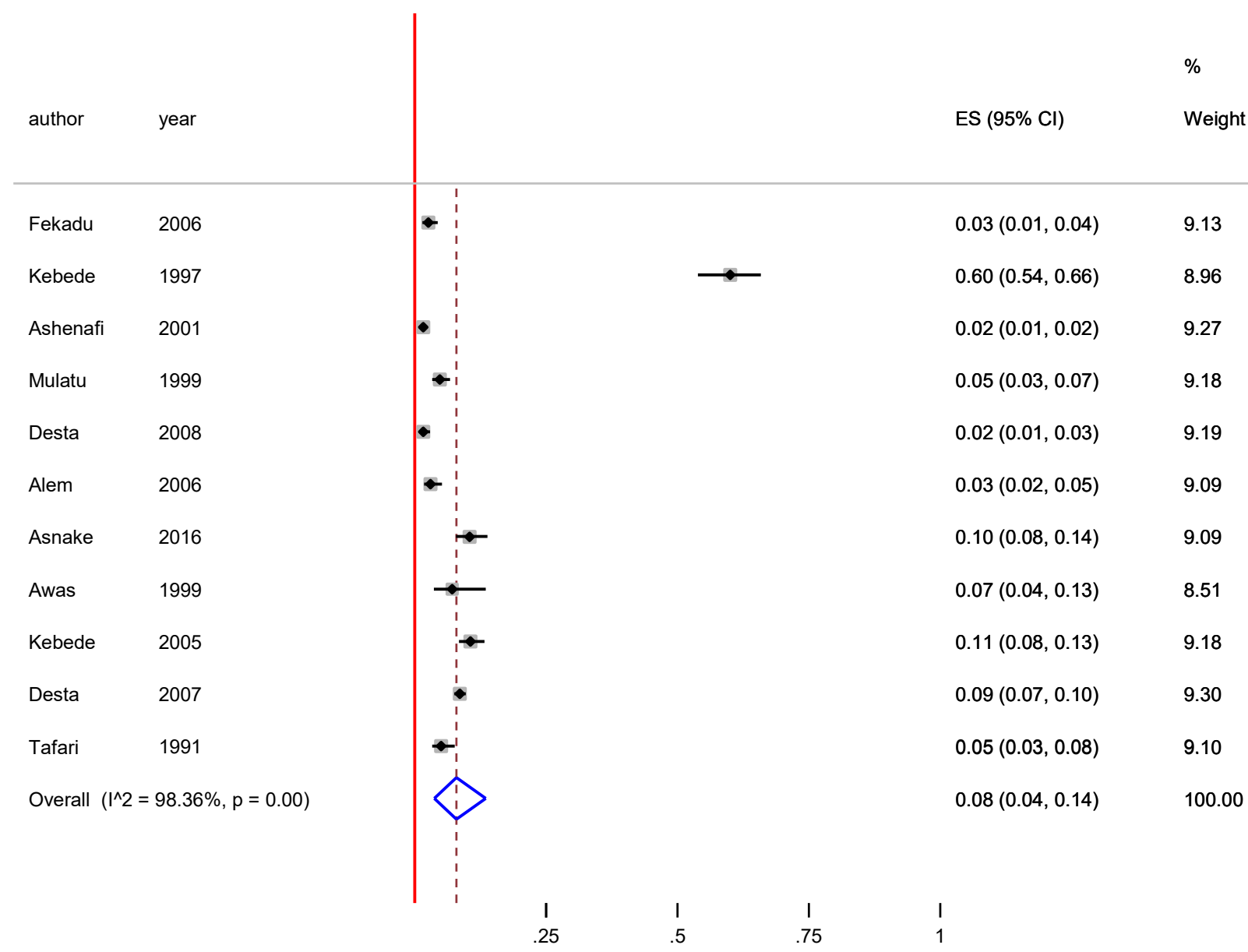

Figure 1: Forest plot presenting prevalence of anxiety using random effect models with $95 \% \mathrm{Cl}$. 


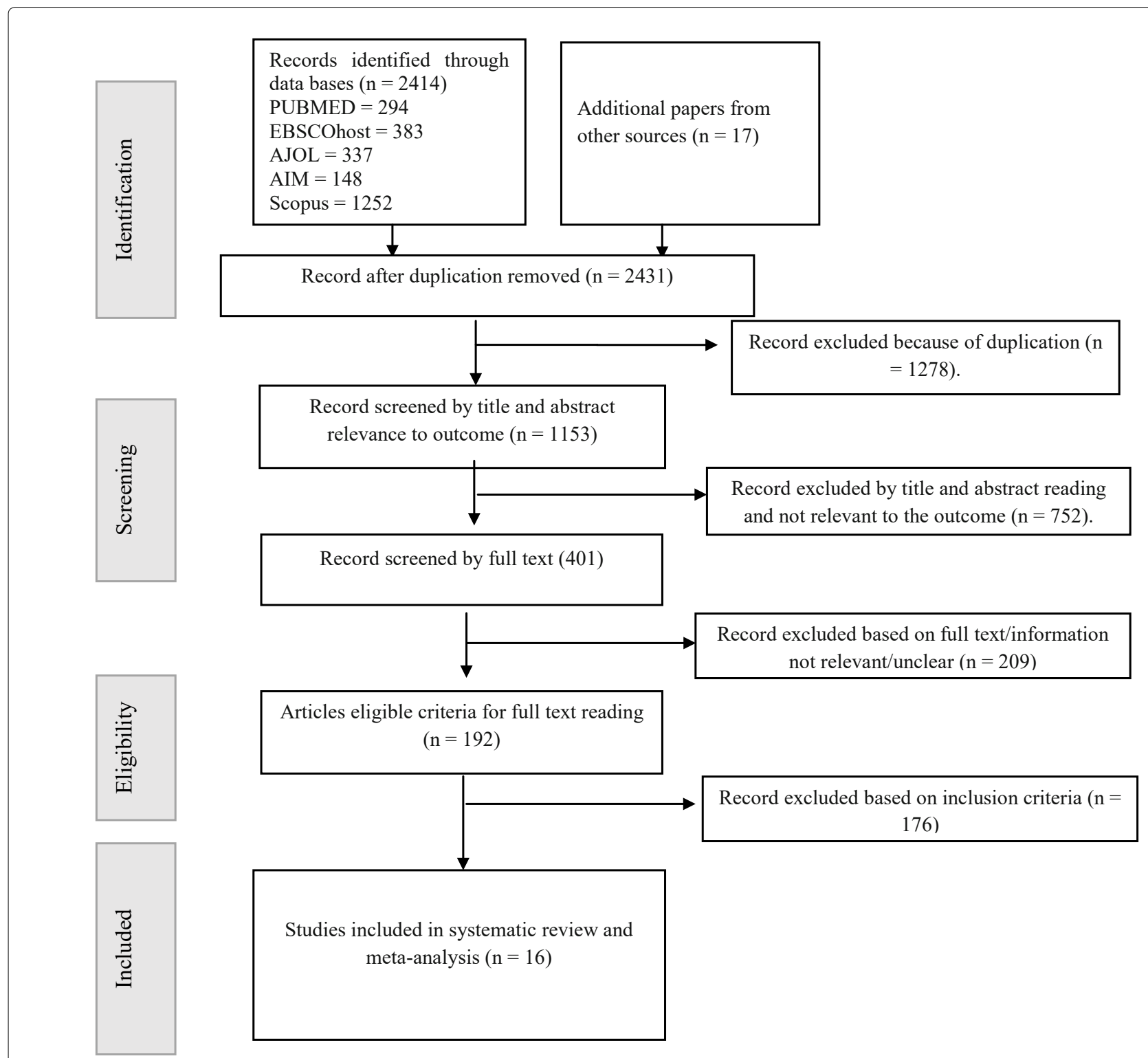

Figure 2: Flow diagram of included studies.

Compared with systematic review and meta-analysis in other countries, the pooled prevalence of anxiety [ $8 \%(95 \% \mathrm{Cl}$ : $4 \%$ to $14 \%$ )] in this study is in line number of community based systematic review and meta-analysis conducted in every region of the world with ranged pooled prevalence of $6.5 \%$ to $10 \%$ for any anxiety disorders in children and youth $[4,10,17,18]$. A recently publish large scale national survey across 82 countries $(N=275,057)$ also estimated a pooled prevalence of $9 \%$ ( $7 \%-12 \%)$, ranging from $8 \%(8-9 \%)$ in Europe to $17 \%$ (16-17\%) in East Mediterranean. This study showed the prevalence of $13 \%$ (13-14\%) in African, 9\% (9$10 \%)$ in America, 8\% (8-9\%) in West Pacific and 7\% (7-8\%) in Asia [18]. Another systematic review and meta-analysis also reported similar results from LMICs (8-27\%), and Iran $[2,47]$. Though, not specific to anxiety, a number of literature reviews in child and adolescents psychiatric disorders were reported [1,2,], in developing country with overall pooled prevalence of $6.46 \%(6.08 \%-6.88 \%)$ in India [48] and $14.3 \%$ $(95 \% \mathrm{Cl}, 13.6 \%-15.0 \%)$ in Sub-Sahara countries [49]. The observed prevalence in the current studies supported the results of previous studies across cultures $[1,2,5,7,10]$. The systematic review that tried to identify the contribution of culture across countries reported similar studies $[7,17,18]$. This study suggested the reasons for the heterogeneity may attributed to methodological and existence of varies risk factors across the world. These various risk factors include: Psychodynamic, psychoanalytic, behavioral, cognitive, genetic and biological also known as Bio-Psycho-Social and no single unifying etiology factor across the world $[2-4,7,12,15]$. In line with this, stress, financial pressure, poverty, political tensions, human rights violations, vulnerability to sexual and reproductive ill-health are common in Ethiopia and reported as risk for anxiety.

Regarding the types and prevalence of anxiety, six different types of anxiety disorders/symptoms [GAD, phobia, social phobia, separation anxiety, OCD and PTSD] were identified. Of these, GAD is the most frequently reported $(n=7)$ anxiety. The estimated pooled prevalence of GAD, phobia, social 
Citation: Bifftu BB, Guracho YD (2021) A Systematic Review and Meta-Analysis of Anxiety among Children and Youth in Ethiopia. J Depress Anxiety Disord 3(2):89-100

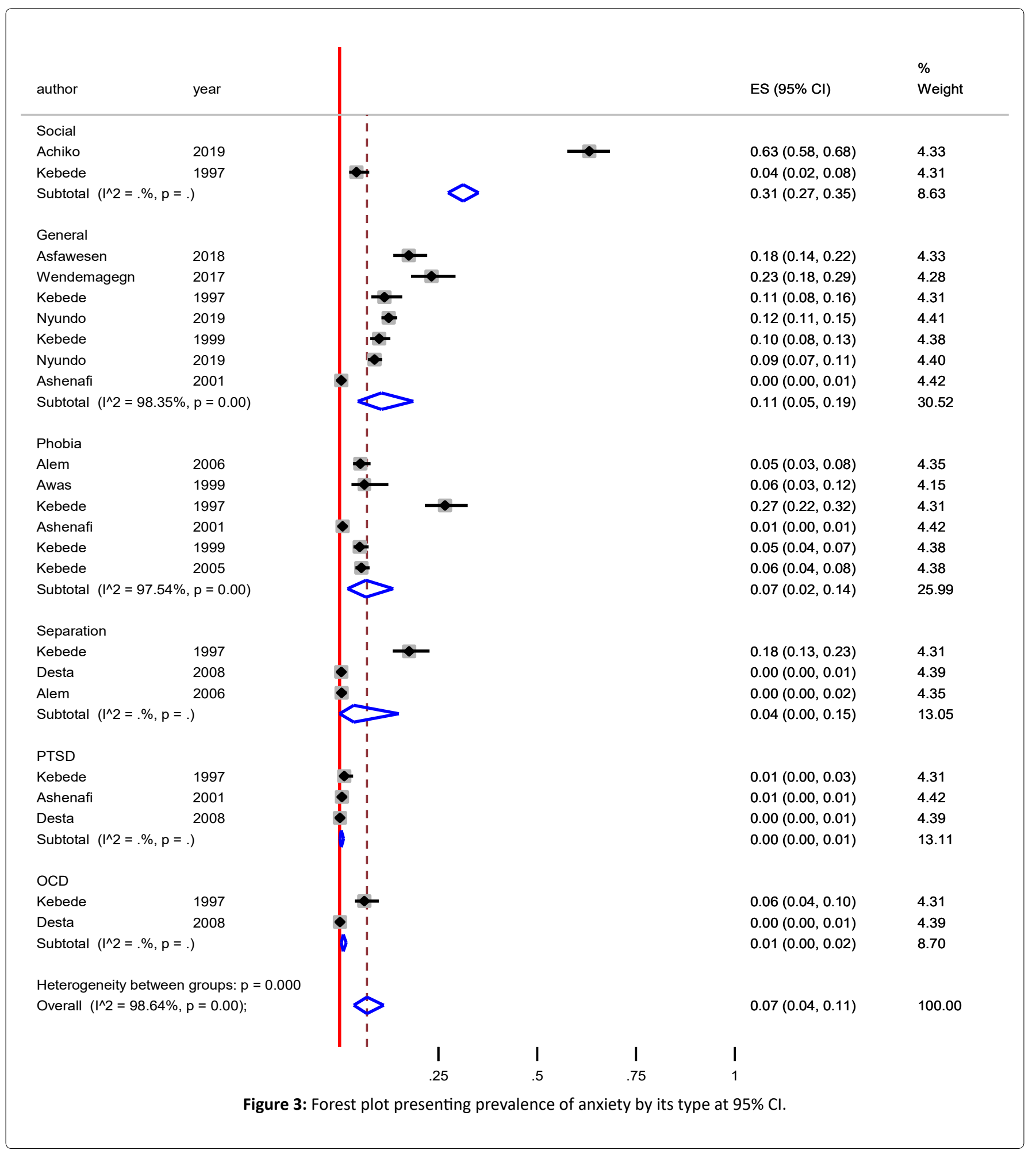



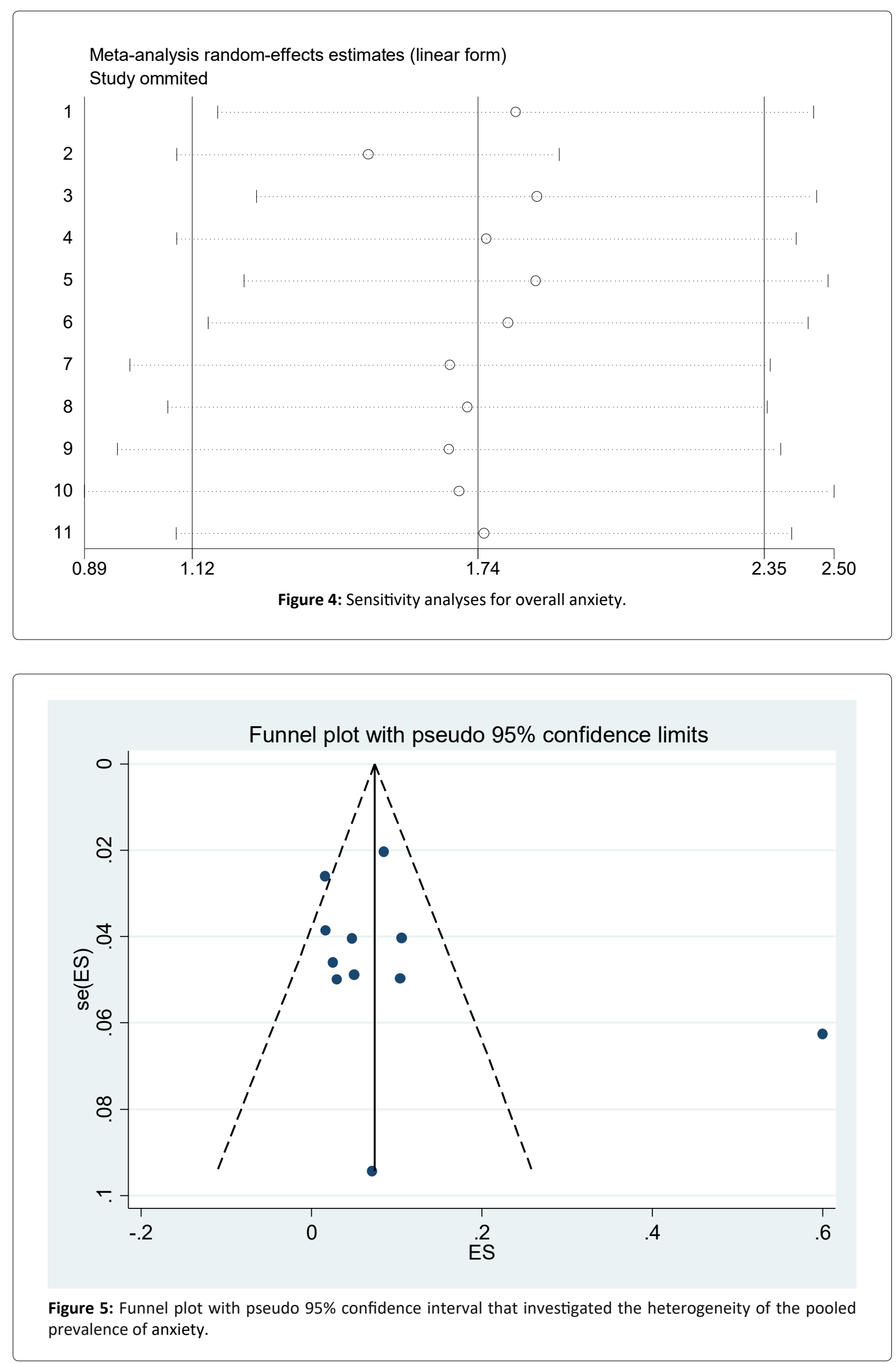
Citation: Bifftu BB, Guracho YD (2021) A Systematic Review and Meta-Analysis of Anxiety among Children and Youth in Ethiopia. J Depress Anxiety Disord 3(2):89-100

phobia, separation anxiety, OCD and PTSD was $11 \%[95 \% \mathrm{Cl}$ : 5-19\%], 7\% [95\% Cl: 2-14\%], 31\% [95\%Cl: 27-35\%], 4\% [95\%Cl: $0-15 \%], 1 \%$ [95\% Cl: 0-2\%] and $0 \%$ [95\% Cl: 0-2\%] respectively. This is consistent with other study $[8,9,16,22]$. The low prevalence of OCD and PTSD in comparison with phobias, social anxiety, and separation anxiety may be attributed to its late age of onset. Evidence showed that phobias, social anxiety, and separation anxiety, have median ages of onset is range $5-10$ years; while, generalized anxiety disorder, panic disorder and posttraumatic stress disorder have median age of onset of 24-50 [7,16,50].

Therefore, observed prevalence of anxiety in this study, disproof the previous notion of mental illness as a problem of adult or some parts of the world and proof the universality of mental illness across all age and countries. Thus, the findings of this study implies: (i) The needs of screening and treatment of children and youth mental health, (ii) The needs of improving support from family, peer group and higher officials, (iii) The needs of evaluating the effectiveness of the national mental health strategy in addressing children and youth mental health care in terms of accessibility, availability and affordability and (iv) Higher officials are also suggested to strengthen mental health awareness.

\section{Strengths and limitations of the study}

This is the first systematic review and meta-analysis about the prevalence of anxiety among children and youth in Ethiopia and use of reference lists and Google Scholar to include all the available studies. However, limitations like exclusion of clinical patient, university students, prisoner, use of different assessment tool, and the observed heterogeneity may limit the representativeness of the study.

\section{Conclusion}

Overall, one in twelve individual in the general populations of children and youth experienced anxiety. This suggests the needs of community mental health screening for children and youth. Children and young people are suggested to strengthen their coping mechanism to stressors. Family and peer groups are suggested to strengthen their support. In addition to this, the results of this finding suggest the needs of evaluating the effectiveness of the national mental health strategy in addressing the mental health of children and youth. Further research is needed to identify contextual risk and protective factors for mental health promotion, prevention and treatment.

\section{References}

1. Polanczyk GV, Salum AV, Sugaya LS, et al. (2015) Annual research review: A meta-analysis of the worldwide prevalence of mental disorders in children and adolescents. J Child Psychol Psychiatry 56: 345-365.

2. Costello EJ, Copeland W, Angold A (2011) Trends in psychopathology across the adolescent years: What changes when children become adolescents, and when adolescents become adults? J Child Psychol Psychiatry 52: 1015-1025.

3. Yatham S, Sivathasan S, Rosalia Y, et al. (2018) Depression, anxiety, and Post-traumatic stress disorder among youth in low And middle income countries: A review of prevalence And treatment interventions. Asian J Psychiatr 38: 78-91.
4. Baxter AJ, Charlson FJ, Somerville AJ, et al. (2011) Mental disorders as risk factors: Assessing the evidence for the Global Burden of Disease Study. BMC Med 9: 134.

5. Kieling C, Henningham HB, Belfer M, et al. (2011) Child and adolescent mental health worldwide: Evidence for action. Lancet 378: 1515-1525.

6. Association AP (2000) Diagnostic and statistical manual of mental disorders. Fourth Edition Revised (DSM-IV).

7. Bandelow B, Michaelis S (2015) Epidemiology of anxiety disorders in the 21st century. Dialogues Clin Neurosci 17: 327335.

8. Beesdo K, Pine DS, Lieb R, et al. (2010) Incidence and risk patterns of anxiety and depressive disorders and categorization of generalized anxiety disorder. Arch Gen Psychiatry 67: 47-57.

9. Beesdo K, Knappe S, Pine DS (2009) Anxiety and anxiety disorders in children and adolescents: Developmental issues and implications for DSM-V. Psychiatr Clin North Am 32: 483-524.

10. Baxter AJ, Scott SK, Vos T, et al. (2013) Global prevalence of anxiety disorders: A systematic review and meta-regression. Psychol Med 43: 897-910.

11. (2017) Global Health Estimates. World Health Organization, Geneva.

12. Somers JM, Goldner EM, Waraich P, et al. (2006) Prevalence and incidence studies of anxiety disorders: A systematic review of the literature. Can J Psychiatry 51: 100-113.

13. Guo X, Meng Z, Huang G, et al. (2016) Meta-analysis of the prevalence of anxiety disorders in mainland China from 2000 to 2015. Sci Rep 6: 28033.

14. Kessler RC, Ruscio AM, Shear K, et al. (2010) Epidemiology of anxiety disorders. Curr Top Behav Neurosci 2: 21-35.

15. Kessler RC, Matthias A, Anthony JC, et al. (2007) Lifetime prevalence and age-of-onset distributions of mental disorders in the World Health Organization's World Mental Health Survey Initiative. World Psychiatry 6: 168-176.

16. Kessler RC, Berglund P, Demler O, et al. (2005) Lifetime prevalence and age-of-onset distributions of DSM-IV disorders in the National Comorbidity Survey Replication. Arch Gen Psychiatry 62: 593-602.

17. Baxter AJ, Vos T, Scott KM, et al. (2010) The regional distribution of anxiety disorders: implications for the Global Burden of Disease Study. Int J Methods Psychiatr Res 23: 422-438.

18. Biswas T, Scott JG, Munir K, et al. (2020) Global variation in the prevalence of suicidal ideation, anxiety and their correlates among adolescents: A population based study of 82 countries. EClinical Medicine 24: 100395.

19. Links PS (1983) Community surveys of the prevalence of childhood psychiatric disorders: A review. Child Dev 54: 531-548.

20. (2010) World Health Organization mental health GAP (WHOmhGAP) intervention guide for mental, neurological and substance use disorders in non-specialized health settings: Version 1.0. World Health Organization, Geneva.

21. Health FDRoEMo (2012) National mental health strategy 2012/13 - 2015/16. Federal Democratic Republic of Ethiopia Ministry of Health, Addis Ababa, Ethiopia.

22. Merikangas KR, He JP, Burstein M, et al. (2010) Lifetime prevalence of mental disorders in US adolescents: Results from the National Comorbidity Survey Replication - Adolescent Supplement (NCS-A). J Am Acad Child Adolesc Psychiatry 49: 980-989. 
23. Erskine HE, Baxter AJ, Patton G, et al. (2017) The global coverage of prevalence data for mental disorders in children and adolescents. Epidemiol Psychiatr Sci 26: 395-402.

24. Ashenafi $Y$, Kebede D, Desta M, et al. (2001) A Prevalence of mental and behavioural disorders in Ethiopian children. East Afr Med J 78: 308-311.

25. Megerssa K, Derege K, Menelik D, et al. (1997) Evaluation of the amharic version of the Diagnostic Interview for Children and Adolescents (DICA-R) in Addis Ababa. Addis ababa university school of graduate studie, 1-66.

26. Alem A, Kebede D, Woldesemiat G, et al. (1999) The prevalence and socio-demographic correlates of mental distress in Butajira, Ethiopia. Acta Psychiatr Scand Suppl 397: 48-55.

27. Awas M, Kebede D, Alem A (1999) Major mental disorders in Butajira, Southern Ethiopia. Artu Psychiutr Rand 100: 56-64.

28. Tafari S, Aboud FE, Larson CP (1991) Determinants of mental illness in a Rural Ethiopian adult population. Sot Scr Med 32: 197-201.

29. Achiko AG, Shikuro EH (2019) Social anxiety disorder among children at gofermeda sub city, Hosanna Town, Ethiopia: Prevalence and associated factors. Psychology 10: 1526-1541.

30. Wondemagegn AT, Cheme MC, Kibret KT (2017) Perceived Psychological, Economic, and Social Impact of Khat Chewing among Adolescents and Adults in Nekemte Town, East Welega Zone, West Ethiopia. Hindawi BioMed Research International 2017: 7427892.

31. Desta M (2008) Epidemiology of child psychiatric disorders in Addis Ababa, Ethiopia. Umea University medical dissertations, Sweden, 1-69.

32. Mulatu MS (1995) Prevalence and risk factors of psychopathology in Ethiopian Children. J Am Acad Child Adolesc Psychiatry 34: 100-109.

33. Fekadu D, Alem A, Hagglof B (2006) The prevalence of mental health problems in Ethiopian child laborers. J Child Psychol Psychiatry 47: 954-959.

34. Kebede D, Alem A (1999) Major mental disorders in Addis Ababa, Ethiopia. III. Neurotic and somatoform disorders. Acta Psychiatrica Scand Suppl 397: 24-29.

35. Kebede D, Fekadu A, Alem A, et al. (2005) The distributions of mental disorder among isolated island community in Southern Ethiopia. Ethiop Med J 43: 71-77.
36. Liranso GS, Mohan SS (2018) Barriers in accessing children's mental health care services in Ethiopia. Prim Health Care 8: 288.

37. Ministry of Youth SC. Federal democratic republic of Ethiopia. Addis Ababa, Ethiopia.

38. Haile K, Chaka A, Ayano G, et al. (2017) Model of decentralization and integration of mental health into primary health care a supplement to the mhGAP program. Austin Med Sci 2: 1017.

39. Shamseer L, David M, Clarke M, et al. (2015) Preferred reporting items for systematic review and meta-analysis protocols (PRISMA-P) 2015: Elaboration and explanation. BMJ 349: g7647.

40. (1989) Convention on the rights of the child. United Nations.

41. Newcastle-Ottawa Scale adapted for cross-sectional studies.

42. Berkey CS, Hoaglin DC, Mosteller F, et al. (1995) A randomeffects regression model for meta-analysis. Stat Med 14: 395411.

43. Higgins JPT, Thompson SG (2002) Quantifying heterogeneity in a meta-analysis. Stat Med 21: 1539-1558.

44. Duval S, Tweedie R (2000) Trim and fill: A simple funnel-plotbased method of testing and adjusting for publication bias in meta-analysis. Biometrics 56: 455-463.

45. Liu J LY (2011) The role of the funnel plot in detecting publication and related biases in meta-analysis. Evidence-based dentistry 12: 121-122.

46. Egger M, Smith DG, Schneider M, et al. (1997) Bias in metaanalysis detected by a simple, graphical test. BMJ 315: 629-634.

47. Zarafshan $H$, Mohammadi M-R, Salmanian M (2015) Prevalence of anxiety disorders among children and adolescents in Iran. A systematic review. Iran J Psychiatry 10: 1-7.

48. Malhotra S, Patra BN (2014) Prevalence of child and adolescent psychiatric disorders in India: A systematic review and metaanalysis. Child Adolesc Psychiatry Ment Health 8: 22.

49. Rao W-W, Xu D-D, Cao X-L, et al. (2019) Prevalence of depressive symptoms in children and adolescents in China: A meta-analysis of observational studies. Psychiatry Res 272: 790-796.

50. de Lijster JM, Dierckx B, Utens EMWJ, et al. (2017) The age of onset of anxiety disorders: A meta-analysis. Can J Psychiatry 62: 237-246. 
PRISMA Checklist: Prevalence of anxiety in children and youth in Ethiopia.

\begin{tabular}{|c|c|c|c|}
\hline Section/topic & $\#$ & Checklist item & $\begin{array}{l}\text { Reported on } \\
\text { page \# }\end{array}$ \\
\hline \multicolumn{4}{|l|}{ TITLE } \\
\hline Title & 1 & Identify the report as a systematic review, meta-analysis, or both. & 1 \\
\hline \multicolumn{4}{|l|}{ ABSTRACT } \\
\hline Structured summary & 2 & $\begin{array}{l}\text { Provide a structured summary including, as applicable: background; objectives; data sources; study } \\
\text { eligibility criteria, participants, and interventions; study appraisal and synthesis methods; results; } \\
\text { limitations; conclusions and implications of key findings; systematic review registration number. }\end{array}$ & 2 \\
\hline \multicolumn{4}{|l|}{ INTRODUCTION } \\
\hline Rationale & 3 & Describe the rationale for the review in the context of what is already known. & $4-5$ \\
\hline Objectives & 4 & $\begin{array}{l}\text { Provide an explicit statement of questions being addressed with reference to participants, } \\
\text { interventions, comparisons, outcomes, and study design (PICOS). }\end{array}$ & 5 \\
\hline \multicolumn{4}{|l|}{ METHODS } \\
\hline Protocol and registration & 5 & $\begin{array}{l}\text { Indicate if a review protocol exists, if and where it can be accessed (e.g., Web address), and, if available, } \\
\text { provide registration information including registration number. }\end{array}$ & NA \\
\hline Eligibility criteria & 6 & $\begin{array}{l}\text { Specify study characteristics (e.g., PICOS, length of follow-up) and report characteristics (e.g., years } \\
\text { considered, language, publication status) used as criteria for eligibility, giving rationale. }\end{array}$ & $7-8$ \\
\hline Information sources & 7 & $\begin{array}{l}\text { Describe all information sources (e.g., databases with dates of coverage, contact with study authors to } \\
\text { identify additional studies) in the search and date last searched. }\end{array}$ & 5 \\
\hline Search & 8 & $\begin{array}{l}\text { Present full electronic search strategy for at least one database, including any limits used, such that it } \\
\text { could be repeated. }\end{array}$ & $5-6$ \\
\hline Study selection & 9 & $\begin{array}{l}\text { State the process for selecting studies (i.e., screening, eligibility, included in systematic review, and, if } \\
\text { applicable, included in the meta-analysis). }\end{array}$ & 6 \\
\hline Data collection process & 10 & $\begin{array}{l}\text { Describe method of data extraction from reports (e.g., piloted forms, independently, in duplicate) and } \\
\text { any processes for obtaining and confirming data from investigators. }\end{array}$ & $7-8$ \\
\hline Data items & 11 & $\begin{array}{l}\text { List and define all variables for which data were sought (e.g., PICOS, funding sources) and any } \\
\text { assumptions and simplifications made. }\end{array}$ & $7-8$ \\
\hline $\begin{array}{l}\text { Risk of bias in individual } \\
\text { studies }\end{array}$ & 12 & $\begin{array}{l}\text { Describe methods used for assessing risk of bias of individual studies (including specification of whether this } \\
\text { was done at the study or outcome level), and how this information is to be used in any data synthesis. }\end{array}$ & $7-8$ \\
\hline Summary measures & 13 & State the principal summary measures (e.g., risk ratio, difference in means). & 8 \\
\hline Synthesis of results & 14 & $\begin{array}{l}\text { Describe the methods of handling data and combining results of studies, if done, including measures of } \\
\text { consistency }\left(\text { e.g., }\left.\right|^{2}\right) \text { for each meta-analysis. }\end{array}$ & 8 \\
\hline
\end{tabular}

\begin{tabular}{|c|c|c|c|}
\hline Section/topic & \# & Checklist item & $\begin{array}{l}\text { Reported on } \\
\text { page \# }\end{array}$ \\
\hline Risk of bias across studies & 15 & $\begin{array}{l}\text { Specify any assessment of risk of bias that may affect the cumulative evidence (e.g., publication bias, } \\
\text { selective reporting within studies). }\end{array}$ & 8 \\
\hline Additional analyses & 16 & $\begin{array}{l}\text { Describe methods of additional analyses (e.g., sensitivity or subgroup analyses, meta-regression), if } \\
\text { done, indicating which were pre-specified. }\end{array}$ & 8 \\
\hline \multicolumn{4}{|l|}{ RESULTS } \\
\hline Study selection & 17 & $\begin{array}{l}\text { Give numbers of studies screened, assessed for eligibility, and included in the review, with reasons for } \\
\text { exclusions at each stage, ideally with a flow diagram. }\end{array}$ & $9 \& 16$ \\
\hline Study characteristics & 18 & $\begin{array}{l}\text { For each study, present characteristics for which data were extracted (e.g., study size, PICOS, follow-up } \\
\text { period) and provide the citations. }\end{array}$ & $9 \& 20$ \\
\hline Risk of bias within studies & 19 & Present data on risk of bias of each study and, if available, any outcome level assessment (see item 12). & $10 \& 17-21$ \\
\hline Results of individual studies & 20 & $\begin{array}{l}\text { For all outcomes considered (benefits or harms), present, for each study: (a) simple summary data for } \\
\text { each intervention group (b) effect estimates and confidence intervals, ideally with a forest plot. }\end{array}$ & $10 \& 17$ \\
\hline Synthesis of results & 21 & Present results of each meta-analysis done, including confidence intervals and measures of consistency. & $10 \& 17$ \\
\hline Risk of bias across studies & 22 & Present results of any assessment of risk of bias across studies (see Item 15). & $17-21$ \\
\hline Additional analysis & 23 & $\begin{array}{l}\text { Give results of additional analyses, if done (e.g., sensitivity or subgroup analyses, meta-regression [see } \\
\text { Item 16]). }\end{array}$ & $10 \& 17$ \\
\hline \multicolumn{4}{|l|}{ DISCUSSION } \\
\hline Summary of evidence & 24 & $\begin{array}{l}\text { Summarize the main findings including the strength of evidence for each main outcome; consider their } \\
\text { relevance to key groups (e.g., healthcare providers, users, and policy makers). }\end{array}$ & $11-13$ \\
\hline Limitations & 25 & $\begin{array}{l}\text { Discuss limitations at study and outcome level (e.g., risk of bias), and at review-level (e.g., incomplete } \\
\text { retrieval of identified research, reporting bias). }\end{array}$ & 13 \\
\hline Conclusions & 26 & $\begin{array}{l}\text { Provide a general interpretation of the results in the context of other evidence, and implications for } \\
\text { future research. }\end{array}$ & 13 \\
\hline \multicolumn{4}{|l|}{ FUNDING } \\
\hline Funding & 27 & $\begin{array}{l}\text { Describe sources of funding for the systematic review and other support (e.g., supply of data); role of } \\
\text { funders for the systematic review. }\end{array}$ & 14 \\
\hline
\end{tabular}

From: Moher D, Liberati A, TetzlaffJ, Altman DG, The PRISMA Group (2009). Preferred Reporting Items for Systematic Reviews and Meta-Analyses: The PRISMA Statement. PLoS Med 6(7): e1000097. doi:10.1371/journal.pmed1000097

For more information, visit: www.prisma-statement.org. 